\title{
Organizaciones sociales afrodescendientes en el municipio de Florencia (Colombia): logros, dificultades y retos
}

\author{
Verenice Sánchez-Castillo ${ }^{* *}$, Carlos Alberto Gómez-Cano ${ }^{* *}$, \\ Yady Melissa Moreno-González ${ }^{\star * * *}$
}

* Artículo de investigación

** Ingeniera Agroecóloga, M.Sc. en Ambiente y Desarrollo, Estudiante de Doctorado en Antropología. Docente Programa Ingeniería Agroecológica de la Universidad de la Amazonia. Grupo de Investigación en Agroecología y Desarrollo Rural (GIADER). Correo electrónico: ve.sanchez@udla.edu.co

*** Contador Público, Administrador Público, Especialista en Pedagogía y en Gestión Pública, estudiante de Maestría en Ciencias de la Educación, docente del Programa de Contaduría Pública de la Universidad de la Amazonia. Grupo de Estudios de Futuro en el Mundo Amazónico (GEMA).

Correo electrónico: carlosgomez325@gmail.com

**** Administradora Financiera, miembro de la Asociación Colonia ChocoanaAcolchoc.

Correo electrónico:

y-melissa27@hotmail.com

Recibido: 30 de julio del 2016 Aprobado: 22 de enero del 2017

Cómo citar este artículo: Sánchez-Castillo, V., Gómez-Cano, C. A., Moreno-González, Y. M. (2017). Organizaciones sociales afrodescendientes en el municipio de Florencia (Colombia): logros, dificultades y retos.

\section{Resumen}

Introducción: la organización de los afros en el municipio de Florencia, Caquetá (Colombia), representa la necesidad de la unión para lograr un reconocimiento y la lucha por sus derechos. Si bien a juicio de los entrevistados la discriminación racial en el departamento es poca, la necesidad de lograr una visibilización y un reconocimiento ha hecho que tres organizaciones de afros se hayan conformado y se sigan consolidando a través del tiempo. El interés del presente manuscrito es precisamente sistematizar estas tres experiencias organizativas a partir de su historia, los logros, las dificultades y los principales retos que enfrentan como colectivo. Metodología: el trabajo es eminentemente cualitativo, la producción de los datos se hizo a partir de instrumentos tales como las entrevistas y la técnica de arqueo de archivos. Resultados: dentro de los principales hallazgos se tiene que las organizaciones existentes son resultado de fragmentaciones internas de la primera, pero que tienen objetivos similares; las mayores dificultades que afrontan están relacionadas con los pocos ingresos para su sostenimiento y poder ayudar más a sus socios. Conclusiones: los logros de las organizaciones afros han sido el reconocimiento por parte del Ministerio del Interior, el posicionamiento en la sociedad caqueteña y que los hayan hecho partícipes de la toma de decisiones de tipo regional. Las dificultades continúan relacionadas con los pocos recursos económicos disponibles y el acceso a proyectos de inclusión socioeconómica rural y urbana. Además, la falta de articulación interna, ya que en algunas ocasiones, a su juicio, prima el bien particular sobre el del grupo. Las tres organizaciones objeto del estudio coincidieron.

Palabras clave: derechos, mejoramiento, organización, reconocimiento. 


\title{
Afro-descendant social organizations in the municipality of Florencia (Colombia): Achievements, difficulties and challenges
}

\begin{abstract}
Introduction: Afro-descendant organization in the municipality of Florencia, Caquetá (Colombia), evidences the need to unify in order to achieve recognition and overcome the struggle for their rights. Although the interviewed subjects declare that there is little racial discrimination in the department, the necessity to attain visibility and recognition has lead to the formation of three Afro-descendant organizations, as well as to their continued consolidation over time. The interest of this manuscript is precisely to systematize these three organizational experiences based on their history, achievements, difficulties and the main challenges they face as a group. Methodology: The work is eminently qualitative. Data were obtained using instruments such as interviews and documentary review. Results: Within the main findings, it is evident that the existing organizations result from internal fragmentation of the organization that appeared fist. Nevertheless, all of them have similar objectives. The biggest difficulties they face relate to the scarce income available for their sustenance and for offering more supporting to their partners. Conclusions: The Afro-descendant organizations' achievements include having been recognized by the Ministry of Internal Affairs, positioning themselves in the Caquetá society and having been taken in account for regional decision making. The difficulties faced continue to be related to the limited economic resources available and tough access to rural and urban socio-economic inclusion projects. The lack of internal articulation must also be mentioned, since the interviewed subjects believe particular interests in some occasions prevail over group objectives and benefits. The three organizations under study agreed in these points.
\end{abstract}

Keywords: rights, improvement, organization, recognition

\section{Organizações sociais afrodescendentes no município de Florencia (Colômbia): conquistas, dificuldades e desafios}

\begin{abstract}
Introdução: a organização dos afrodescendentes no município de Florencia, Caquetá (Colômbia), representa a necessidade da união para atingir um reconhecimento e da luta por seus direitos. Embora, na opinião dos entrevistados, o preconceito racial no estado seja pouco, a necessidade de atingir uma visibilização e um reconhecimento fez com que três organizações de afrodescendentes tenham se conformado e continuem se consolidando. $\mathrm{O}$ interesse deste texto é precisamente sistematizar essas três experiências organizacionais a partir de sua história, conquistas, dificuldades e principais desafios que enfrentam como coletivo. Metodologia: o trabalho é eminentemente qualitativo; a produção dos dados se deu a partir de instrumentos como as entrevistas e a técnica de análise de arquivos. Resultados: um dos principais achados se refere a que as organizações existentes são resultado de fragmentações internas da primeira, mas que têm objetivos semelhantes; as maiores dificuldades que enfrentam estão relacionadas com os poucos ingressos para sua conservação e com poder ajudar mais seus sócios. Conclusões: as conquistas das organizações afrodescendentes são o reconhecimento por parte do Ministério do Interior, o posicionamento na sociedade de Caquetá e a participação na tomada de decisões de tipo regional. As dificuldades continuam relacionadas com os poucos recursos econômicos disponíveis e o acesso a projetos de inclusão socioeconômica rural e urbana. Além disso, a falta de articulação interna, já que, em algumas ocasiões, no seu julgamento, prima o bem particular sobre o do grupo. As três organizações objeto do estudo coincidiram.
\end{abstract}

Palavras-chave: direitos, melhoramento, organização, reconhecimento. 


\section{Introducción}

\section{Antecedentes latinoamericanos organizativos de los afrodescendientes}

Los afros que llegaron al continente americano lo hicieron por la oleada de violencia que trajo consigo el proceso de sometimiento a la esclavitud. Es así como en 1519, un primer grupo de afros desembarcó en Puerto Rico, y desde entonces la esclavitud negra representó uno de los principales pilares económicos en América y en Europa (Duconge y Lube, 2014).

Las injusticias ocasionadas a los afros, así como los malos tratos dados por sus amos durante el tiempo de la esclavitud, hizo que hacia el siglo XviI, en Haití, se generara su primera revolución, la cual posteriormente generó una ola de rebeliones (LaoMontes, 2009).

Así las cosas, bajo la influencia de los movimientos afros de América del Norte y los movimientos panafricanistas europeos en la década de los cincuenta y la de los sesenta fueron surgiendo las organizaciones afrodescendientes en Latinoamérica. Una de las organizaciones formadas en este movimiento fue la afroecuatoriana, fundada por jóvenes intelectuales influenciados por las lecciones del marxismo y del movimiento literario panafricano en Quito. Su primer avance fue la creación del Centro de Estudios Afroecuatorianos, el cual actuó como una escuela de pensamiento y análisis de las circunstancias que rodeaban al mundo africano y afroamericano (LaoMontes, 2009). Durante esta década, los esfuerzos de los pueblos afroamericanos por consolidar la unidad transcontinental dieron frutos ideológicos notables, los cuales se expusieron en el Primer Congreso de la Cultura Negra de las Américas, desarrollado en Cali (Colombia), en agosto de 1977 (Sánchez, 2003). En el mismo año se conformó la organización ProcoodesUnión Provincial de Cooperativas de Pescadores de Esmeraldas en Ecuador, con 2500 pescadores del Cantón y 11000 en toda la Provincia. En esta se integró una perspectiva diferente a la tradicional y se articuló la herencia pesquera del negro a la organización social (Gómez, 2006).

Posteriormente, en 1995 se instauró la Red de Organizaciones Negras Centroamericanas (ONECA), la cual, en línea con las anteriores, buscaba promover el desarrollo integral de los pueblos afros desde la perspectiva de los derechos humanos. En el 2000 se conformó la Alianza Estratégica Afrodescendiente de América Latina y el Caribe, realizada con el fin de discutir la participación de las comunidades de 29 organizaciones afros en la conferencia de Durban del 2001. Esto sirvió como espacio organizativo y pedagógico para la formación y consolidación de las redes afros que se empezaron a abrir espacios propios en la región (Buffa y Becerra, 2006).

Seguidamente, y como resultado de esta conferencia, los diferentes gobiernos y organizaciones mundiales entendieron que lo afro implicaba la adopción de criterios culturales y no meramente biológicos, en camino hacia un autoreconocimiento (Buffa y Becerra, 2006). En la misma dimensión, algunos eventos de carácter mundial siguieron promulgando el respeto por los derechos y la identificación de un espacio en el cual coordinar las políticas específicas para los afros y elevarlas a los respectivos gobiernos nacionales, entre ellos vale mencionar: los objetivos de desarrollo del milenio (ONU, 2000), la Conferencia Mundial contra el Racismo, Discriminación Racial, Xenofobia y Formas Conexas de Discriminación (Organización de las Naciones Unidas-ONU, 2001), y el Parlamento Negro de las Américas (2005).

\section{Los afrodescendientes en Colombia, su lucha social y algunos de sus logros}

En Colombia los primeros registros sobre la llegada de los afros se remontan, al igual que en América, a la época de la colonia, cuando los grandes hacendados los utilizaban como mano de obra esclava para trabajar en la explotación de materias primas tales como el algodón, el azúcar, el arroz y el tabaco, entre otros, y en algunos casos en las minas. Arribaban legalmente al país por el puerto de Cartagena de Indias, en donde eran distribuidos hacia el interior del país a través de los ríos Cauca y Magdalena. No obstante, otros lugares como Popayán, Santa fe de Antioquia, Honda, Anserma, Zaragoza y Cali se constituyeron en centros secundarios de comercio (España et al., 2012). Por otra parte, su ingreso de forma ilegal se hizo por el Litoral Pacífico en el puerto de Buenaventura, Charambirá y Gorgona, y por el Atlántico en el puerto de las costas de Riohacha, Santa Marta, Tolú y el Darién (Corredor, 2003).

Las luchas sociales y las estrategias organizativas de los afrodescendientes datan desde cuando estaban sometidos a la esclavitud, pues por la forma en que ingresaron al país fueron víctimas de tratos inhumanos, de humillaciones y de exclusión por parte de la sociedad, la cual no los reconocía como personas 
libres y con derechos (Ngou-Mve, 1997; Serbin, 1991). En vista de esto, este grupo de personas empezaron a considerar "la organización" como su válvula de escape a todas las situaciones de anomia social a las que eran sometidos, al tiempo que era su forma de rechazo a la esclavitud. Es de esta manera como aparecen los cimarrones, una forma organizativa conformada por afrodescendientes que rechazaban la esclavitud y que, gracias al espacio colectivo, el grupo podía mantener sus prácticas religiosas, crear sus propios espacios culturales, hacer reclamos legales y producir recursos para comprar su libertad (Lao-Montes, 2009).

Los cimarrones escapaban de sus amos para internarse en los palenques y luchar por la búsqueda de la libertad de su pueblo. Este grupo creó su propio estilo de vida, basado en sus costumbres, bailes, música y su propio lenguaje. Sus gobiernos consistían en autoridades escogidas dentro de la comunidad y desde allí organizaban los ataques contra los esclavistas (Escalante, 2005; Wabgou, 2012).

Producto de esta gran, sangrienta y no corta ni fácil lucha, se logró el reconocimiento de los derechos de los afrodescendientes en el país, a partir de la promulgación de la Ley de la Libertad que abolió legalmente la esclavitud y la esclavización de personas en Colombia. Esta ley se dio en 1851 bajo el mandato del presidente José Hilario López. Sin embargo, los cimarrones y sus descendientes quedaron en el país como ocupantes del territorio, pero de manera ilegal, por haber sido excluidos del ordenamiento jurídico republicano y del Estado de derecho, e ignorados en las leyes como sujetos jurídicos con derechos étnicos y como ciudadanos especiales (Mosquera, 2001).

Así las cosas, solo fue en 1991 con la reforma a la Constitución Política de Colombia que las comunidades afros asentadas en el Pacífico, organizadas en los aspectos étnico territoriales, lucharon por el reconocimiento de la comunidad como grupo étnico y lograron su cometido, con una ventana de posibilidad concreta al derecho a la titulación de tierras colectivas que se había venido luchando desde la década de los ochenta (Hoffman, 2002; Wabgou, 2012). A partir de la reforma a la Constitución se promulgaron leyes y decretos tales como los que se describen a continuación.

La Ley 70 de 1993, por la cual se desarrolla el artículo transitorio 55 de la Constitución Política, en el que se establecen los "derechos colectivos sobre el territorio a las comunidades negras, asentadas en las tierras baldías, rurales y ribereñas de la cuenca del pacifico y otras zonas del país".
El Decreto 1745 del 12 de octubre de 1995, en el que se reglamentó el capítulo inI de la Ley 7, en el cual se destaca cómo:

Una comunidad negra podrá constituirse en Consejo Comunitario, que como persona jurídica ejerce la máxima autoridad de administración interna dentro de las Tierras de las Comunidades Negras, de acuerdo con los mandatos constitucionales y legales que lo rigen y los demás que le asigne el sistema de derecho propio de cada comunidad.

El Decreto 1371 de 1994, que en su artículo 45 señala:

El Gobierno Nacional creará la Comisión Consultiva de Alto Nivel con la participación de representantes de las Comunidades Negras de Antioquia, Valle, Cauca, Chocó, Nariño, Costa Atlántica, Raizales de San Andrés y Providencia, Santa Catalina y demás regiones a que se refiere la misma ley.

Asimismo, en los Artículos 48, 66 y 67 del mismo decreto se define la participación de los miembros de las comunidades negras en espacios públicos como la Cámara de Representantes, el Consejo Nacional de Planeación y el Consejo de Política Económica y Social.

Por otra parte, en la línea educativa, en 1996 se promulgó el Decreto 1627, mediante el cual se creó un fondo especial de créditos condonables para los estudiantes de comunidades negras. De igual forma, en los aspectos políticos de representación y participación, la Ley 649 les permitió a los afros tener dos cupos en las curules del congreso (Buffa y Becerra, 2006).

\section{La organización afro en Colombia}

Si bien, como se dijo anteriormente, los antecedentes organizativos de los afros están mucho antes de la Constitución de 1991, con todo el proceso del cimarronaje, sin duda alguna la reforma a la Ley 70 , la cual considera "que el Estado apoyará mediante la destinación de los recursos necesarios, los procesos organizativos de las comunidades negras con el fin de recuperar, preservar y desarrollar su identidad cultural", dinamizó en alguna medida este proceso. Para ello, se creó la Dirección de Asuntos para las Comunidades Negras, asignada al Ministerio del Interior, la cual tiene como objetivo ejercer las competencias de la Secretaría Técnica de la Comisión Consultiva de Alto Nivel y fortalecer el sentido de solidaridad y el proceso organizativo de las Comunidades Negras mediante 
la creación y actualización del Registro Único de Organizaciones de Base de las Comunidades Negras (Ministerio del Interior, 2016; Quintero, 2010).

Según la información recopilada por Quintero (2010), los departamentos que presentan la mayor participación de organizaciones son aquellos en los cuales el peso relativo de la población afrocolombiana es mayor, los mismos en los cuales se han aplicado con mayor fuerza las políticas públicas y la adjudicación de tierras colectivas. Se trata de la mayoría de los departamentos del Pacífico (Chocó, Cauca, Valle y Nariño), además del departamento de Bolívar, el cual presenta gran proporción de población afrocolombiana (el 65\% de las organizaciones oficialmente registradas se concentra en estos cinco departamentos).

Sin embargo, aunque distribución poblacional afrocolombiana se centró inicialmente en la zona del Pacífico y Bolívar, no obstante, por diferentes causas tales como la colonización dirigida y la guerra al interior del país, parte de este grupo migró hacia otros territorios de la nación como la Baja Bota Caucana, Putumayo y Caquetá (Martínez, 2011).

\section{Los afros en el departamento del Caquetá}

La presencia del afrocolombiano en el departamento del Caquetá obedece a diferentes procesos: en un primer momento, corresponde a los negros del Pacífico nariñense, quienes en la década del cincuenta, oriundos de Barbacoas, Maguí y Payán, deciden partir hacia territorio del río Caquetá, pasando por Pasto, Valle del Sibundoy y Mocoa, hasta llegar a Puerto Limón en el Putumayo, y luego al Caquetá por el puerto del río Caquetá, punto de entrada a un nuevo imaginario cultural. El segundo momento corresponde a la población afrodescendiente proveniente del Cauca en la década de los setenta y la de los ochenta, y en una menor cantidad del Chocó, quienes huían de sus tierras debido a la violencia generada en el país (Carrillo, 2014).

El tercer proceso de migración se evidencia a partir de la década del setenta y corresponde a un alto número de docentes del Chocó que motivados por la posibilidad de trabajo, llegan al Caquetá y los nombran en diferentes municipios, pues en tiempos del apogeo de la coca ser maestro no era un atractivo en la región. El motivo entonces de este nuevo desplazamiento era la búsqueda de empleo, ocasionado, además, por la carencia de docentes en el departamento (Corpoamazonia, 2015).
Según el DANE (2015), la población afro asentada en Caquetá asciende a 11661 personas, una cifra no tan significativa en el panorama nacional, ni en la relación del total de individuos pobladores del Caquetá. Esta situación, a juicio de los afros, ha hecho muchas veces se les intente desconocer en los beneficios que estos pobladores tienen a nivel nacional. Es por ello que se ha considerado de importancia estratégica formalizar los grupos de organización afro en el departamento.

\section{La organización afro en el departamento, a manera de introducción}

La primera organización afro conformada en el departamento fue la Asociación de la Colonia Chocoana del Caquetá (ACOLCHOC), integrada por docentes que llegaron al Caquetá en el tercer momento descrito anteriormente. Ellos traían sus familias, en busca de mejorar su calidad de vida (CORPOAMAZONIA, 2015).

En la actualidad, se han logrado constituir con Alcolchoc, 12 organizaciones de base en todo el departamento: la Fundación para la Difusión de la Cultura Negra (Fundine); la Fundación Afrodescendiente del municipio de Curillo y sur de Colombia (FACURI), en el municipio de Curillo; la Fundación de Afrocolombianos Unidos por la Cultura y los Derechos Humanos (FunAmu); la Asociación Afro amazónica (AsofaC), en Florencia; La Organización Afro Colombiana Luther King zona urbana; la Organización Tío Tom; y la Organización Afrocolombiana Construyendo Territorio, en Puerto Rico; la Asociación de Negritudes de San Vicente del Caguán (anersan), en San Vicente del Caguán; Niches en Acción, en Valparaíso; Barack Obama, en Solita; y la Organización de Comunidades Malcom, en El Doncello.

Dado lo expuesto, se tiene que, si bien la población afrodescendiente ha logrado la conformación de importantes asociaciones - doce en el departamento-, en su mayoría urbanas, se desconoce cuál ha sido la dinámica organizativa de estas, las razones y las causas que motivaron su conformación, su estado actual, sus movimientos y si aún continúan con luchas sociales por el reconocimiento o hacia dónde están orientados sus intereses. Es por esto que el presente ejercicio de investigación realiza una aproximación a las organizaciones urbanas afros del municipio de Florencia, también como un pedido de estás mismas a través de uno de sus líderes a fin de sistematizar sus experiencias. En este sentido, se busca no solo 
conocer la historia organizativa, sino abordar sus retos, intereses y principales lecciones aprendidas hasta el momento, y cómo estas han influenciado al grupo social al que pertenece cada organización.

\section{Metodología}

La presente investigación se desarrolló en el municipio de Florencia, departamento del Caquetá, localizado al suroccidente de la República de Colombia. Las organizaciones consultadas corresponden a formas organizativas afros que residen en la ciudad en el casco urbano y hace algún tiempo desarrollan sus procesos organizativos.

El enfoque metodológico es histórico hermenéutico, pues la realidad es una construcción que el investigador hace de un pedazo de esta, de manera que en ella interesa el proceso, más que los resultados mismos del proceso (Bonilla y Rodríguez, 1999; Gutiérrez, 2014).

El diseño metodológico es de tipo cualitativo, pues no consta de un solo método sino de variaciones de métodos, y comprende múltiples realidades que reflejan bases históricas, con una visión del mundo con diversidad de interpretaciones (Gómez, Sánchez y Torres, 2016). Cabe mencionar que este tipo de diseños se aplican con el propósito de construir conceptos y relaciones en los datos brutos y luego organizarlos en un esquema explicativo teórico. En este orden de ideas, se hace una aproximación global a las situaciones sociales con el fin de explorarlas, describirlas y comprenderlas de manera inductiva. Es decir, a partir de los conocimientos que tienen las diferentes personas involucradas en ellas, y no deductivamente, con base en hipótesis formuladas por el investigador externo.

El proceso del trabajo inicia con la petición de una estudiante afrodescendiente, quien expresa que su comunidad desea sistematizar su ejercicio organizativo y, con esto, la intención de que esta líder local colabore en el proceso. Este interés se expandió posteriormente a todas las organizaciones afro de Florencia, las cuales fueron invitadas a participar del ejercicio con una gran aceptación.

El método de trabajo tuvo dos fases, las cuales se desarrollaron de manera simultánea: una fue la consulta en fuentes tales como documentos, informes, boletines y planes acerca de la organización afro en el departamento, y luego en la ciudad. Para ello se empleó la técnica de arqueo de archivo, mediante la cual, apoyados por una plantilla de doble entrada, se enlistaron los documentos hallados, se escribió un resumen acerca del hallazgo y se tomó nota de los posibles temas que apoyarían los documentos.

La otra fase que iba avanzando era la de campo, la cual inició con la identificación de actores clave. De esta manera, por cada organización se establecieron los respectivos contactos, teniendo en cuenta que para el desarrollo del trabajo se requería la persona más antigua en la organización o que conociera todo el proceso.

Posteriormente, se diseñó una entrevista semiestructurada, en la que se abordaron las variables en tres momentos: historia-inicios, actualidad-logros y dificultades y futuro-retos. Las entrevistas fueron grabadas, previa autorización de los entrevistados. La información obtenida con las entrevistas fue analizada mediante códigos y categorías de análisis, en el marco del protocolo propuesto por Bonilla y Rodríguez (1997), así: descripción de los hallazgos aislados, identificación de relaciones entre variables, formulación de tentativas entre los fenómenos, revisión de los datos en búsqueda de evidencias que corroboren o invaliden los supuestos que guían el trabajo, formulación de explicaciones sobre el fenómeno e identificación de esquemas teóricos más amplios que contextualicen el patrón cultural identificado y, finalmente, se redactaron los hallazgos.

\section{Resultados y discusión}

En Colombia, la reforma a la Constitución Política y los diferentes soportes jurídicos que se han establecido en defensa de los derechos y el reconocimiento de las comunidades afros les ha permitido avanzar en su desarrollo social. Aun así, de acuerdo con Caicedo y Castillo (2008), las problemáticas de discriminación, violencia y exclusión en contra de las comunidades afrodescendientes continúan hasta la fecha.

Para Antón, Bello, del Pololo, Paixao y Rangel (2009), los afrodescendientes presentan problemáticas sociales relacionadas con la discriminación y la exclusión, y señalan prácticas racistas que históricamente han ocurrido en el país, las cuales, además, empiezan con la intención de perpetuar la discriminación racial a través del lenguaje y terminan en la arraigada exclusión de los mercados laborales que experimentan a diario miles de afrocolombianos (Reales, 2005). Dicho sea de paso, situaciones tales como viviendas inadecuadas, ausencia de servicios públicos, economías insubsistentes, ausencia de programas de desarrollo comunitarios y de inversión social, y falta de trabajo, entre otras, han contribuido 
al deterioro de los grupos sociales de esta comunidad (Quiroz, Felizzola y Villareal, 2014).

No obstante, pareciera esto un panorama general en toda la población afrocolombiana, de manera que conviene señalar cómo las problemáticas difieren un poco, dependiendo del lugar en que habiten los implicados. Por ejemplo, los habitantes del Pacífico enfrentan situaciones por la lucha del territorio contra las multinacionales, mientras que los que se acentúan en las cabeceras municipales se la juegan por la denuncia de la discriminación racial y la lucha por el acceso a condiciones de mejoramiento de calidad de vida (PNUD, 2010).

Así las cosas, y con el ánimo de hacer frente de manera especial a los problemas de exclusión, en Florencia se encuentran tres organizaciones afrocolombianas, cuya experiencia alrededor de la lucha social no ha sido fácil, y en este trasegar han tenido logros, pero también dificultades, así como algunos retos por enfrentar.

La presente investigación abordó de manera juiciosa los discursos de estas tres organizaciones. Sus conversaciones y testimonios permitieron agrupar su ejercicio de liderazgo en tres momentos - que para el caso juegan como categorías de análisis-: inicios, actualidad y retos futuros. También permiten hacer una aproximación a las mayores dificultades que han tenido que enfrentar y las motivaciones que los mantienen para continuar.

\section{Momento 1: construíamos sueños}

\section{Asociación de la Colonia Chocoana del Caquetá-Alcochoc}

De entrada, el nombre de la asociación pone sobre la mesa un asunto que marca identidad, pues de acuerdo con Quintero (2010), las colonias, si bien son asociaciones informales construidas con los lazos de origen social y espacial, pueden generar procesos de consolidación más formales, como es el caso de Alcochoc. Además, agrega el autor, los nombres de las organizaciones hacen alusión, por lo general, a un municipio, al ser esta una construcción identitaria de tipo local en la ciudad en la que se establecen unas fronteras simbólicas que unen con el sitio de origen, al mismo tiempo que con la ciudad donde se establecen (Quintero, 2010).

Acolchoc se creó en 1992, de modo que hoy cuenta con 24 años de existencia. Se encuentran próximos a celebrar sus bodas de plata con cerca de 400 asociados, y recuerdan que su mayor motivación para conformar la asociación fue "participar de manera activa en el festival de las colonias" (J. A. Cañada, comunicación personal, noviembre 5 del 2015). Inicialmente, se conformaron como un Comité, pero pronto, y al percibir los diferentes procesos por el posicionamiento afro que se venían dando a nivel nacional, luego de la Constitución Política de 1991, decidieron conformar Alcolchoc, ya "no como organización de tipo folclórico o cultural, sino una organización para luchar por el bienestar de la comunidad afro" (V. Pino, comunicación personal, octubre 28 del 2015).

Para Rossbach (2009) la dinámica social de la constituyente y de la legislación siguiente, fomentó la unificación legal de los afros, lo cual, a su juicio, ocurrió con la perspectiva de obtener las ventajas de una discriminación positiva, tales como lograr derechos territoriales seguros y colectivos y obtener participación política. Esta situación significa, en la opinión de los juristas, una indemnización por las injusticias cometidas contra la población negra y sus antepasados (Barbary, 2001). En la misma línea, la Conferencia Nacional de Organizaciones Afrocolombianas (CNAO), sostiene que las organizaciones Étnico Territoriales de los Campesinos Negros de los ríos del Pacífico, conformadas para la defensa de su territorio contra las multinacionales, se fortalecieron precisamente con la reforma a la Constitución Política del 1991 (CNAO, 2015). Desde este enfoque, Baquero (2014) agrega que la reforma a la Constitución Política, en la que se reconoció el carácter multiétnico de la nación y se prohibieron las manifestaciones de discriminación racial, protege a los afrodescendientes aun por fuera de las comunidades ribereñas, como a cualquier colombiano.

Por su parte, Mínguez (2015) se refiere a la participación de los afros en política, pues la organización es un escenario político de participación a través del cual se pueden generar resultados que apuntan a la necesidad de diseñar y aplicar un marco normativo legal que garantice la supervivencia de las expresiones culturales y de organización de dichos colectivos en sus territorios.

\section{Asociación Afroamazónica del Caquetá (ASOFAC)}

Trece años después de conformada ALCOLCHOc, además dado el aumento de la población afro en Florencia, y motivados por dos asuntos concretos 
-abrirse espacios propios y luchar por sus derechos-, otro grupo de afros decidió conformar un nuevo colectivo organizado: ASOFAC, conformado por cerca de 45 familias. Uno de sus representantes considera que, en primera medida, no se sentían lo suficientemente representados por la otra asociación y, por tanto, no tenían acceso a los beneficios:

El señor declaró que se vio la necesidad de una asociación con sentido regional y como habíamos otro grupo de Afros que queríamos abrirnos espacios por la poca cabida que teníamos en las otras organizaciones; también por nosotros, por luchar por nuestros propios intereses... las otras, pues no digamos que eran excluyentes sino que era un círculo un poco cerrado. (D. Perea, comunicación personal, noviembre 5 de 2015)

Esta motivación de la visibilización es algo frecuente en las organizaciones afros, pues se considera que a partir de ahí se generan otras dinámicas que les permiten estar más de cerca de las discusiones en los niveles de gobierno, por ejemplo, en torno a la política pública y el enfoque diferencial. Este es el claro ejemplo de la Asociación de Afrodescendientes Desplazados (Afrodes), quienes manifiestan que la organización les ha permitido generar sus propios espacios y poner en conocimiento las situaciones que viven las poblaciones afros en medio del conflicto armado, y de igual forma les ha permitido tratar con el gobierno políticas públicas para la comunidad (AFRODES, 2016).

\section{Fundación Afrocolombianos Unidos por la Cultura y los Derechos Humanos (Funamo)}

Dos años más tarde de conformada Asofac, un grupo de mujeres afros también consideraron que no podían acceder fácilmente a los beneficios por parte de las organizaciones existentes. Lo anterior, aunado a que su participación activa en el Programa Familias en Acción les permitió conocer experiencias organizativas de mujeres afros en diferentes partes del país tales como el Bajo Magdalena, Medellín y el Cauca, con excelentes resultados, lo que las motivó a organizarse como colectivo; en este caso el objetivo de mayor peso fue la organización con el fin de hacer defender sus derechos. De esta manera lo recuerda una de sus fundadoras, la sra. Rodríguez: "Viendo las experiencias de otras partes, les pregunté, ¿por qué no hacemos una fundación o algo para que nosotras podamos defender nuestros derechos?, y empezó a trabajar la idea y ya fue cuando creamos la fundación Funamu" (L. Rodríguez, comunicación personal, noviembre 30 de 2016).

Al respecto, Abregú (2007) resalta cómo, más allá de una lucha por los derechos en términos de acción-reacción, las organizaciones que luchan por los derechos humanos y la ciudadanía han venido trabajando en forma cada vez más sistemática en torno a la incorporación de la perspectiva de derechos en un escenario de mayor relevancia como lo son las políticas públicas, conscientes de que solo este tipo de acciones les permitirán maximizar los resultados de su esfuerzo en el propósito de alcanzar a un universo más amplio y más diverso de la sociedad.

El tema familiar es un aspecto central, tanto en Funamo, como en su actualidad el trabajo en familia y dignificar el papel de la mujer como eje articulador. Para Camacho (2004), el desarraigo y la deshumanización causada por la esclavitud, hace que de manera tradicional la familia como centro social y evolutivo adquiera importancia para la reconstrucción social de grupos, de la familia y del parentesco.

Además de lo anterior, aparece el flagelo del desplazamiento y la pérdida de las costumbres afros, como su principal consecuencia y, por tanto, la necesidad del rescate cultural: "La Funamu surge porque nosotros como negros siempre seremos doblemente, yo diría triplemente revictimizado" (A. R., Quiñonez, comunicación personal, noviembre 27 del 2015).

Al respecto, la Consultoría para los Derechos Humano y el Desplazamiento (CodHes), menciona que el pueblo afrocolombiano es uno de los sectores sociales más afectados por el desplazamiento y el desarraigo social, cultural, económico y territorial. Uno de los factores que caracterizan este desarraigo se da por la falta de capacidad institucional para hacerlo evidente. Un claro ejemplo de esta situación son las bajas cifras de desplazamiento forzado de la población afrocolombiana consignadas en el Registro Único de Población Desplazada (RUPD), las cuales revelan que solo el $10 \%$ del total de la población afro ha sufrido este flagelo, lo que demuestra niveles de afectación doble en proporción a su estatuto en la estructura demográfica nacional y los altos niveles de subregistro, así como de los deficientes procesos de reconocimiento étnico por parte de las instituciones estatales (CODHEs, 2013). Estar organizados, para el caso de Funamu, se concibe como una forma de enfrentarse a este flagelo.

Por otra parte, tanto en las motivaciones para organizarse de Asocaf, como de Funamo, se puede leer en el discurso de las entrevistadas "la no 
representación" y "el no sentirse beneficiados". Al respecto, Rodríguez (2014) considera que este es precisamente uno de los detonantes de los conflictos dentro de una organización, el cual se fundamenta, principalmente, en que estos suceden cuando dos o más intereses se encuentran en estado activo y los dos buscan su realización y perfeccionamiento. En ese contexto, los intereses propios se enfrentan a los intereses opuestos y cada uno busca prevalecer sobre el otro. El efecto previsible de esta acción — porque en su estado activo los intereses conducen precisamente a la acción-, solo puede conducir a un resultado: un interés se impone sobre el otro, un interés prevalece y el otro no. En esta lógica resulta muy difícil entender estados intermedios o resultados parciales. A fin de establecer que un conflicto en particular ha concluido, se necesita también establecer qué interés ha prevalecido y qué interés ha desaparecido. Para Lucaya, Dalmau y Ferrer (2015), el asunto que genera conflicto también puede ser relacional, en este caso de las personas en la organización - por ejemplo, el apoyo social entre iguales-, y esto produce una bifurcación del grupo social conformado.

Finalmente, en este caso en particular, es importante resaltar el papel del agente externo para dinamizar la conformación de la organización, que en este caso pareciera ser de manera indirecta. Sin embargo, Lemus (2009) considera que los procesos organizativos de las mujeres afros son más escasos y que su organización es reciente y coincide con las intervenciones de programas gubernamentales para el "desarrollo", con la cooperación internacional o con ONG locales, desde la década de los ochenta.

Por otra parte, si bien se reconoce aún bastante discriminación racial afro, lo es en mayor medida al sexo femenino. Para Camacho (2004), las mujeres afros a lo largo de la historia han estado envueltas en un contexto de poder patriarcal, dominación colonial, violencia, desplazamiento y fragmentación que se mantiene hasta el día de hoy. Igualmente, las mujeres están en medio de la lucha por la supervivencia y la liberación, pues en los espacios públicos y los privados ellas han trabajado por distintos medios para ser sujetos activos. Sin embargo, aun así, están marcadas por los prejuicios existentes en las instituciones, las organizaciones negras y en la academia alrededor de los temas concernientes a la raza, la etnicidad, la mujer y el género, de ahí que su voz, sus palabras y, en general, su presencia, estén tan ausentes de los textos escritos y de la vida pública:
Ha sido un proceso, y tiene un sacrificio largo que me ha costado lágrimas, desprecio, humillación, desprecio de diferentes instituciones e índoles, pero a Dios gracias fortaleza y apoyo de algunas personas, que creyeron en mis ideas la fundación está funcionando (A. R., Quiñonez, comunicación personal, noviembre 27 de 2016)

\section{Momento 2: ¡Así estamos!}

A nivel departamental, a través de la gestión de Acolchoc se logró tener acceso a la Oficina de Asuntos Étnicos, la cual se divide en dos secciones: una para los indígenas, y una oficina de asuntos étnicos para los afrocolombianos. La Secretaría de Asuntos Étnicos es la que orienta, dirige y coordina las acciones enmarcadas dentro de la nación colombiana para el reconocimiento de los grupos étnicos asentados en Colombia como colectivos sociales con identidad propia y se ubica en las principales cabeceras (Gobernación del Valle del Cauca, 2016).

Para Asofac, sus principales logros están relacionados con la apertura de espacios y la obtención de una visibilización del afro en el Caquetá. Esto se ve reflejado en que ya los convocan como asociación a diferentes espacios que por ley tienen derecho a nivel departamental, tales como los conPEs y los consejos departamentales y municipales de planeación.

En el caso de FUNAMU, su mayor logro lo concentran en la creación de unidades productivas, pues a través de la Organización Internacional para las Migraciones (OIM), se logró emplear muchas afros en cuestiones de modistería. A la fecha, Funamo no es el ejecutorio directo, sino que recibe y le pasa a Mujeres Constructoras de Paz; de esta manera, Funamo, ahora con apoyo de la Pastoral Social, recibe el encargo, y se apoya en Mujeres Constructoras de Paz, quienes se responsabilizan de la confección de uniformes de colegio, togas, arreglos para grado, uniformes de dotación, chaquetas, sudaderas y buzos.

La consolidación de distintas propuestas generadas por las organizaciones afros se ha realizado con un trabajo que ha contado con el acompañamiento del ACNur y que se ha construido con el aporte de organizaciones regionales, consejos comunitarios y organizaciones de la sociedad civil. AFRODEs declaró:

Estamos seguros de seguir avanzando en el proceso de articulación en marcha de cerca de 60 procesos organizativos de población afrocolombiana en situación de desplazamiento forzado distribuidos en todo 
el país, que en forma autónoma continúan con sus esfuerzos contribuyendo a la adopción de un enfoque diferencial, de acuerdo con lo establecido por la Corte Constitucional y el gobierno colombiano. (Reina y Román, 2011, p. 10)

\section{Reconocimiento por el Ministerio del Interior}

Las tres organizaciones coinciden en que uno de sus mayores logros es estar reconocidas por el Ministerio del Interior, pues si bien el proceso jurídico de reconocimiento de una organización afro inicia como cualquier grupo organizado, es decir, realización de asamblea para la creación, la creación de estatutos, el nombramiento de junta directiva y la legalización ante la Cámara de Comercio, luego de desarrollar una serie de procedimientos para que la organización, asociación o fundación sea reconocida por el Ministerio, en el caso de Funamo, por ejemplo, esta última parte tardó más de cinco años.

El procedimiento para estar reconocidas se percibe, a juicio de los entrevistados, como un proceso largo, complicado y costoso, pues todos los trámites se realizan en Bogotá. Sin embargo, las tres organizaciones han seguido al pie de la letra lo emanado en el Decreto 3770 de 2008, a fin de llevar a cabo el Registro Único de Organizaciones de las Comunidades Negras, Afrocolombianas, Raizales y Palenqueras y los Consejos Comunitarios. El decreto precisa que solo podrán inscribirse en tal registro aquellas organizaciones que cumplan con los siguientes requisitos: tener dentro de sus objetivos reivindicar y promover los derechos humanos, territoriales, sociales, económicos, culturales, ambientales y/o políticos de las comunidades negras, tener más de un año de haberse conformado como tales, diligenciar el Formulación Único de Registro, el acta de constitución de la organización, y los estatutos de la organización - los cuales obligatoriamente deben tener los capítulos de: estructura interna de la organización, procedimiento para la elección de sus representantes y dignatarios, procedimiento para la toma de decisiones, nombres de sus voceros o representantes elegidos democráticamente, plan de actividades anual y dirección para correspondencia-.

Además, el mencionado decreto precisa en uno de sus parágrafos que en los estatutos de las organizaciones a que alude el presente artículo, se deberá establecer expresamente que las personas que integran la organización deben ser miembros de comunidades negras, afrocolombianas, raizales o palenqueras.
La importancia de estar reconocidas por el Ministerio radica en que, de acuerdo con el artículo 41 de la Ley 70: "el Estado apoyará mediante la destinación de los recursos necesarios, los procesos organizativos de las comunidades negras con el fin de recuperar, preservar y desarrollar su identidad cultural", de manera que obviamente todas buscan estar reconocidas. En este sentido y a fin de dar cumplimiento a la ley, por medio del Decreto 2313 de 1994 se creó la Dirección de Asuntos para las Comunidades Negras, asignada al entonces Ministerio del Interior. En la actualidad, esta oficina se reestructuró en la Dirección de Etnias del ahora Ministerio del Interior y de la Justicia. Esta entidad debe, entre otras competencias, ejercer la Secretaría Técnica de la Comisión Consultiva de Alto Nivel y fortalecer el sentido de solidaridad y el proceso organizativo de las comunidades negras, mediante la creación y actualización del Registro Único de Organizaciones de Base de las Comunidades Negras (Quintero, 2010).

\section{Dificultades}

Para Acolchoc, uno de los asuntos más difíciles en todo este proceso ha sido hacer entender a los gobernantes de turno la responsabilidad que tienen dentro del proceso con las comunidades afrocolombianas, pues como administrativos no conocen la legislación o, en palabras de una de los entrevistados, "se hacen lo que vulgarmente llamamos lo del ojo gordo" (D. Perea, comunicación personal, noviembre 5 de 2015)

De manera similar, Asofac asegura que lo más difícil que han tenido que enfrentar es la falta de oportunidades para los afros, al igual que la falta de espacios, muy a pesar de que constitucionalmente tienen espacios de participación. A su juicio, los gobiernos locales coartan mucho la participación del afro, lo que pone barreras al avance de la organización.

Los indios, los negros y las mujeres, entre otros, han hecho parte de los sectores de población dominados o minorizados desde el poder hegemónico. El poder político dominante los ha representado como minorías y el saber académico los ha asumido como tales. En el mismo sentido, los imaginarios sociales son reflejo y re-producen a la vez formas de relación marcadas por la condición subalterna de estos sectores de la población (Restrepo y Rojas, 2004).

Otra de las dificultades que menciona Asofac se da en relación a lo limitado de los recursos económicos, los desplazamientos a Bogotá para hacer los trámites en el Ministerio del Interior, las filas 
desgastantes, los rechazos, etc. De igual forma, menciona que es difícil lograr la credibilidad con las otras organizaciones y los celos de estas mismas.

Por su parte Funamu resalta que lo más difícil de este proceso ha sido tener contentos a los cooperantes y no dejar que sus intereses los desunan. De igual forma, resaltan que en las diferencias de origen, entre una región y otra, siempre hay visiones diferentes respecto a lo cultural y esto dificulta los procesos, y que infortunadamente la cultura enseña una competencia, pero es individual por lo que a veces resulta difícil crear asociación y pretender que todos se van a beneficiar de la misma manera, o que todos van a trabajar equitativamente, de modo que siempre es complejo el tema de la cooperación.

\section{Momento 3: asumimos nuevos retos}

Para Acolchoc, el reto más importante es el tema de etnoeducación, la lucha para que sus hijos y los hijos de sus compañeros tengan una educación diferencial. En este sentido educativo, también sale a flote la imperiosa necesidad de lograr que la Universidad de la Amazonia entienda el derecho que tiene la comunidad afrocolombiana del orden nacional, y esto se vea reflejado en los pagos de las matrículas y no pasar por tantos trámites sin respuesta concretas.

La etnoeducación aparece como un reto en las diferentes organizaciones, en este sentido, se tiene que este sistema constituye un proceso a través del cual los miembros de un pueblo internalizan y construyen conocimientos y valores y desarrollan habilidades y destrezas de acuerdo con sus características, necesidades, aspiraciones e intereses culturales, que les permiten desempeñarse adecuadamente en su medio y proyectarse con identidad hacia otros grupos humanos (Artunduaga, 1997). Es por lo anterior que la etnoeducación no se refiere exclusivamente a estudios sobre lo afro, sino que se refiere a la creación de nuevos espacios de pensamiento activo, de debate y diálogo, en los que se involucra la historia, la memoria, el dolor y los conocimientos diversos que pueda generar el colectivo (Restrepo y Rojas, 2004).

Ahora bien, de manera particular se tiene que si bien en Colombia se cuenta con un importante desarrollo normativo en materia de educación para grupos étnicos o política etnoeducativa, infortunadamente esto solo se aplica en la educación básica y media. En educación superior no se cuenta con ningún tipo de normatividad que determine la manera como esta se relaciona con la etnoeducación, pues muy a pesar de que se ha avanzado en la apertura de cupos para estudiantes indígenas y afrocolombianos en algunas universidades, no existe una política oficial que regule, no solo la condición de ingreso a los centros de educación superior, sino que facilite las condiciones de permanencia y las reformas curriculares que se requieren para llevar a cabo una verdadera acción afirmativa en la universidades (Caicedo y Castillo, 2008). Esto guarda estrecha relación con uno de los principales retos de Acolchoc en materia educativa, en este caso en el relacionamiento con la Uniamazonia.

Por otra parte, buscan consolidar su sitio de reuniones y un hogar de paso para sus compañeros Afros que tienen que venir a la ciudad por algún trámite y no tienen donde pasar la noche.

Para Funamu, lo importante al mediano plazo es organizar un grupo de personas que trabajen por el rescate de la cultura, crear unidades productivas para las personas y poder subsidiar en algo a los estudiantes o brindar una oportunidad de trabajo. Así, en línea con Acolchoc, buscan tener una casa para la organización, la casa Funamu, donde se quiere que funcione una biblioteca y todo lo relacionado con el rescate cultural de los afros, siendo la organización líder en la región.

Lo anterior, para Oslender (2002), muestra la importancia que representa para los negros la localidad, la cual se refiere no solo a los escenarios físicos dentro de los que ocurre la interacción social, sino que implica también que estos escenarios y contextos se utilicen de manera rutinaria por los actores sociales en sus interacciones y comunicaciones cotidianas. De esta manera, se dejan identificar ciertas localidades como escenarios físicos asociados con las interacciones típicas que componen las colectividades como sistemas sociales.

Según Álvarez (2007), las manifestaciones artísticas son una estrategia para el rescate de la música y la danza en diferentes generaciones, de diferentes intereses que se componen en el escenario, en este caso, la casa.

\section{Conclusiones}

La lucha social afro en el departamento del Caquetá, a diferencia de la del interior del país, que ha estado orientada al logro de territorios colectivos, se ha manifestado principalmente en un esfuerzo por la visibilización y el reconocimiento para estos grupos organizados. Su imaginario de reconocimiento está 
relacionado con el poco interés del Estado por su condición afro, en razón a que el porcentaje de individuos en ese territorio no es representativo.

Si bien en Florencia se identifican tres grupos organizados, estos son el resultado de subdivisiones $\mathrm{y}$ fragmentaciones internas de un grupo madre que aun con objetivos similares y en un mismo territorio, no se sienten lo suficientemente representados.

El imaginario afro alrededor del liderazgo no ha cambiado mucho en las organizaciones a través del tiempo. Tampoco se hace diferenciador o particularizante entre una u otra organización, por tanto, posicionarse y lograr reconocimiento en las áreas políticas, organizativas, culturales y educativas son su mayor muestra de solidez organizativa, pues una organización líder será aquella que desarrolle mejores procesos alrededor de estos temas.

En lo político, el liderazgo es proyectado hacia la confluencia de un mayor número de socios y una mayor cobertura -inicialmente en un escenario local, luego regional y, finalmente, en la consultiva nacional-; dicho sea de paso, esta es una meta que desde los inicios cada organización ha tenido de manera particular.

En lo cultural, la necesidad del rescate de sus costumbres y el traslado de estas desde los ancianos a los pequeños, es un asunto prioritario. Así las cosas, tradición y valores se combinan para que se consolide en mayor medida lo autóctono, lo identitario y, por supuesto, la lucha por la diferencia.

Respecto al proceso educativo, las comunidades afro reconocen la importancia de la formación académica, especialmente la del nivel universitario, pues consideran que esta permite disminuir las brechas sociales, y lograr así el reconocimiento social de sus integrantes. En el mismo sentido, resaltan la importancia de construir una "educación propia" cimentada en su cultura, como una estrategia para la conservación de su identidad como pueblo. Igualmente, son conscientes de que deben unificar sus esfuerzos para exigir a las entidades territoriales, entre ellas la Universidad de la Amazonia, la garantía de sus derechos, logrando de esta manera ser una fuerza viva, activa y dinámica dentro del contexto regional.

Finalmente, desde la dimensión humana y social, las comunidades afro del Caquetá hacen hincapié en la hermandad y solidaridad como un elemento estratégico en sus sistemas organizacionales. Prueba de esto son las grandes preocupaciones en torno a la obtención de infraestructura propia - bienes inmuebles-, que además de facilitar el desarrollo operativo de las organizaciones, permita cobijar a sus coterráneos provenientes de zonas distintas a Florencia. Es así como la obtención de centros operativos multifuncionales es una de los grandes retos y expectativas de las organizaciones

\section{Agradecimientos}

Los investigadores se permiten agradecer a las organizaciones Afrodescendientes del municipio de Florencia Acolchoc, Funamo y Asofac, a todos sus líderes y lideresas y, en especial, a sus directivas y quienes participaron de manera directa en este ejercicio, por permitirnos realizar el presente trabajo y compartirnos sus sentires y experiencias, todo lo cual, con su autorización, hoy podemos compartir a la comunidad académica.

\section{Referencias}

Abregú, M. (2007). Derechos humanos para todos: de la lucha contra el autoritarismo a la construcción de una democracia inclusiva.Puente@Europa, 5(1),6-41

Álvarez, L. (2007). La Casa de la Cultura Indo-Afro-Americana, un espacio de construcción y puesta en juego de identidades. xxvi Congreso de la Asociación Latinoamericana de Sociología. Asociación Latinoamericana de Sociología, Guadalajara, México.

Antón, J., Bello, A., del Pololo, F., Paixao, M. y Rangel, M. (2009). Afrodescendientes en América Latina y el Caribe: del reconocimiento estadístico a la realización de derechos. Recuperado de http://www.cinu.mx/minisitio/ Afrodescendientes/Afrodescendientes.pdf.

Artunduaga, L. (1997). La etnoeducación: una dimension de trabajo para la educación en comunidades de Colombia. Revista Iberoamericana de Educación, 13, 35-46.

Asociación de Afrodescendientes Desplazados-Afrodes. (2016). Afrodes: desplazados afrocolombianos organizados para exigir sus derechos. Recuperado de http:// www.hchr.org.co/afrodescendientes/index.php/portada/afrodes-desplazados-afrocolombianos-organizados-para-exigir-sus-derechos.html

Baquero, P. (2014). Organización política de los afrocolombianos residentes en Bogotá. Revista Hallazgos, 12(23), 275-285.

Barbary, O. (2001, agosto). Identidad y ciudadanía afrocolombiana en Cali y la Región Pacífica: pistas estadísticas para una interpretación sociológica. Revista Electrónica de Geografía y Ciencias Sociales, 22(94), 7-25. 
Bonilla, E. y Rodriguez, P. (1997). Más allá del dilema de los métodos. La investigación en ciencias sociales. Bogotá: Editorial Norma.

Buffa, D. y Becerra, M. (2006). La población afrodescendiente en América Latina y el Caribe. Recuperado de http:// www.cea2.unc.edu.ar/africa-orientemedio/libros/ afrodescendientes/15Diego-Buffa-y-Maria-Jose-Becerra.pdf

Caicedo, J. A. y Castillo, E. (2008). Indígenas y afrodescendientes en la universidad colombiana: nuevos sujetos, viejas estructuras. Cuadernos Interculturales, 10.

Camacho, J. (2004). Silencios elocuentes, voces emergentes: reseña bibliográfica de los estudios sobre la mujer afrocolombiana. En M. Prado, C. Mosquera, y M. Ramírez (Ed.), Panorámica Afrocolombiana: Estudios sociales en el Pacífico, 167-212.

Carrillo, L. (2014). Consecuencias sociales del cultivo de la coca en comunidades afrocolombianas del Caquetá: análisis de la relación entre la economía, las prácticas campesinas tradicionales y su papel en la seguridad alimentaria. Ágora U.S.B, 14(1).

Conferencia Nacional de Organizaciones Afrocolombianas-CNAO. (2015). Historia del Pueblo Afrocolombiano. Recuperado de http://www.convergenciacnoa.org/ documentacion.html

Consultoría para los Derechos Humanos y el Desplazamiento-Codhes. (2013). La crisis humanitaria en Colombia persiste: el Pacífico en disputa. Documentos Codhes, 26.

Corporación para el Desarrollo Sostenible del Sur de la Amazonia-Corpoamazonia. (2015). Plan de desarrolloAfrocaqueteño2015-2025.Florencia,Caquetá: Corpoamazonia.

Corredor, R. (2003). Los afrodescendientes en la historia de Colombia. Recuperado de http://axe-cali.tripod.com/ cepac/hispafrocol/3.htm

DANE. (2015). Demografía y Población. Recuperado de http://www.dane.gov.co/ index.php/estadisticas-por-tema/demografía-y-población

Decreto 1371 de 1994. Por el cual se conforma la comisión consultiva de alto nivel que trata el artículo 45 de la Ley 70 de 1993. Julio 1 de 1994. Do 41417.

Decreto 2313 de 1994. Por el cual se adiciona la estructura interna del Ministerio de Gobierno con la dirección de asuntos para las comunidades negras y se le asignan funciones. Octubre 14 de 1994. Do 41566.
Decreto 1745 de 1995. Por el cual se reglamenta el capítulo III de la Ley 70 de 1993, se adopta el procedimiento para el reconocimiento del derecho de propiedad colectiva de las tierras de las comunidades negras y se dictan otras disposiciones. Octubre 13 de 1995. Do 42049.

Decreto 1627 de 1996. Por el cual se reglamenta el artículo 40 de la Ley 70 de 1993. Septiembre 13 de 1996. Do 42877.

Decreto 3770 de 2008. Por el cual se reglamenta Comisión Consultiva de Alto Nivel de Comunidades Negras, Afrocolombianas, Raizales y Palenqueras; se establecen los requisitos para el Registro de Consejos Comunitarios y Organizaciones de dichas comunidades y se dictan otras disposiciones. Septiembre 25 del 2008. Do 47123.

Duconge, G. I. y Lube, M. (2014). Diásporas, etnicidad y etnogénesis: de las reflexiones teóricas a los estudios de caso sobre las comunidades afro-descendientes en América latina. Papeles de trabajo-Centro de Estudios Interdisciplinarios en Etnolingüística y Antropología Socio-Cultural, 28, 95-119.

Escalante, A. (2005). Palenques en Colombia. Revista CESLA, 7. 385-390.

España, D. Males, N. Marín, L. Pérez, J. Restrepo y I. Álvarez, V. (2012). Afrocolombianidad. Recuperado de http:// es.slideshare.net/danii1308/culturas-afrocolombianas.

Gobernación del Departamento del Cauca. (2016). Secretaría de Asuntos Étnicos. Recuperado de https://www. valledelcauca.gov.co/etnicos/

Gómez, C. A., Sánchez, V. y Torres, L. E. (2016). Análisis del impacto del Programa Jóvenes en Acción de la Universidad de la Amazonia periodo 2014-2015. Revista FACCEA, 6(1), 58-69.

Gómez, C. (2006). Identidades y Políticas Culturales en Esmeraldas y Cali: Estudio De Casos Sobre Organizaciones Afro, Producción Cultural y Raza (tesis de maestría), Maestría en Estudios de la Cultura, Universidad Andina Simón Bolívar, Ecuador.

Gutiérrez, M. (2014). Los enfoques filosóficos de generación del conocimiento y las apuestas metodológicas que exigen. Recuperado de http://www.javeriana.edu.co/ blogs/mlgutierrez/files/Enfoques-y-estrategias-de-investigacion4.pdf

Hoffmann, O. (2002). Conflictos territoriales y territorialidad negra. En Afrodescendientes en América. Bogotá: UN-ICANH-IRD-ILSA. 
Lao-Montes, A. (2009). Movimientos Afroamericanos: contiendas políticas y desafíos históricos. En R. Hoetmer (Ed.), Repensar la política desde América Latina: Cultura, Estado y movimientos sociales (pp. 169-185). Lima.

Ley 70 de 1993. Por la cual se desarrolla el artículo transitorio 55 de la Constitución Política. Agosto 31 de 1993. Do 41013 .

Ley 649 de 2001. Por la cual se reglamenta el artículo 176 de la Constitución Política de Colombia. Marzo 28 de 1993. Do 44371.

Lemus, D. (2009, junio). Mujeres negras/afrocolombianas en los procesos organizativos en Colombia: Un aporte al estado del debate. Reflexión Política, 11(21).

Lucaya, D., Dalmau, I. y Ferrer, R. (2015). Gestión de conflictos en las organizaciones: un enfoque psicosocial integrador. Seguridad y Salud en el Trabajo, 8, 26-32.

Martínez, P. (2011). Población negra, Cultura Afrocolombiana. Buenaventura.

Mínguez, X. (2015). Conflicto y paz en Colombia: Significados en organizaciones defensoras de los derechos humanos. Revista de Paz y Conflicto, 8(1). 179-196.

Ministerio del Interior. (2016). Dirección de Asuntos para Comunidades Negras, Afrocolombianas, Raizales $y$ Palenqueras. Recuperado de http://www.mininterior. gov.co/mision/direccion-de-asuntos-para-comunidades-negras-afrocolombianas-raizales-y-palenqueras

Mosquera, J. (2001). Afrocolombianidad. Boletín del Movimiento Nacional Afrocolombiano Cimarrón. Bogotá.

Ngou-Mve, N. (1997). El cimarronaje como forma de expresión del África bantú en la América colonial: el ejemplo de Yangá en México. México: África Negra.

Organizaciones de las Naciones Unidas-onu. (2000). Objetivos de Desarrollo del Milenio. Recuperado de http:// www.un.org/es/millenniumgoals/bkgd.shtml.

Oslander, U. (2002). Espacio, lugar y movimientos sociales: hacia una espacialidad de resistencia. Revista Electrónica de Geografía y Ciencias Sociales, 6(115), 105-132.

Parlamento Negro. (2005). Constitución del Parlamento negro. Recuperado de http://parlamentonegro.blogspot.com. co/2008/06/constitucin-del-parlamento-negro.html
Organización de las Naciones Unidas-onu. (2001). Conferencia Mundial contra el Racismo, la discriminación Racial, la Xenofobia y las formas Conexas de Intolerancia. Recuperado de http://www.un.org/es/ events/pastevents/cmcr/

Programa de las Naciones Unidas para el Desarrollo-PNUD. (2010). Políticas públicas para el avance de la población afrocolombiana: revisión y análisis. Panamá.

Quintero, O. (2010). Los afros aquí: Dinámicas organizativas e identidades de la población afrocolombiana en Bogotá. Boletín de Antropología Universidad de Antioquia, 24(41), 65-83.

Quiroz, K., Felizzola, V. y Villareal, N. (2014). Proyecto pedagógico afrocolombiano. Recuperado de http://proyectopedagogicoafrocolombiano. blogspot.com.co/2014/06/ proyecto-pedagogic o-afrocolombiano.html

Reales, L. (2005). Racismo y políticas públicas en Colombia. El caso afrocolombiano (1991-2005). Recuperado de http://portail-eip.org/SNC/ EIPColombia/2005/afro_ colombiens/Racismo.pdf

Reina, J. y Román, C. (2011). Organizaciones afrocolombianas lanzan propuesta de decreto sobre ley de víctimas. Recuperado de http://www.acnur.org/ t3/noticias/ noticia/organizaciones-afrocolombianas-lanzan-propuesta-de-decreto-sobre-ley-de-victimas/

Restrepo, E. y Rojas, A. (2004). Conflicto e (in)visibilidad: Retos en los estudios de la gente negra en Colombia. Popayán: Universidad del Cauca.

Rodríguez, J. (2014). La importancia del manejo de conflictos en las organizaciones modernas. Bogotá: Universidad Militar Nueva Granada.

Rossbach, L. (2009). ¿Qué pasa con el Pacífico Negro en el Atlántico Negro?: El Atlántico Negro de Paul Gilroy frente a los acontecimientos (afro) colombianos. Memorias, $22,11-23$.

Sánchez, J. (2003). Diagnóstico de la problemática afroecuatoriana y Propuestas de Acciones Prioritarias. Quito.

Serbin, A. (1991, enero-febrero). ¿Por qué no existe el poder negro en América Latina? Nueva Sociedad, 111.

Wabgou, M. (2012). Herencia Negroafricana en Colombia. Recuperado de http://estudiosafricanos.cea.unc.edu. ar/files/06-Wabgou-N\%C2\%B09.pdf 